\title{
Dietary Calcium Intake and Associated Factors Among Pregnant Women in Loma District, South West Ethiopia, 2020
}

Adisu Hailu Tofu ( $\nabla$ hailuadisu15@gmail.com )

Dawuro Zone Health department https://orcid.org/0000-0001-5998-1200

\section{Yibeltal Kassa}

Wolaita Sodo University, College of Medicine and Health Science, Department of public Health

Damene Darota Amamo

Wolaita Sodo University, College of medicine \& Health Science, Department of Public Health

\section{Research}

Keywords: Dietary calcium intake, pregnant women, Loma district

Posted Date: January 21st, 2021

DOI: https://doi.org/10.21203/rs.3.rs-150326/v1

License: (c) (1) This work is licensed under a Creative Commons Attribution 4.0 International License.

Read Full License 


\section{Abstract}

Background: Low dietary calcium intakes could cause harmful effects to the pregnant woman by influencing pregnancy outcome. Adequate intake of dietary calcium during pregnancy reduces the risk of complications and aids in improved birth outcome. Many researchers focus on urban areas to address the issue and our study was focused on rural community. Hence the study is aimed at assessing dietary calcium intake in rural communities.

Objective: To assess the dietary calcium intakes and associated factors among pregnant women in Loma, south west Ethiopia from May to July, 2019

Methods: -A community based cross-sectional study was conducted in Loma district, Dawuro zone, southwest Ethiopia. Data were collected from 398 pregnant women after random selection from nine kebeles. Simple random sampling technique was employed to select study subjects. Socio-economic and demographic, and health related data was collected using structured, interviewer administered, and pre tested questionnaire. The $24 \mathrm{hr}$ dietary recall was used for three different days with seven days interval as per the recommendation. When dietary data is collected, often it was converted into nutrients, especially the calcium intake by using Ethiopian food composition table. Collected data was checked for completeness, and entered in to Epi Data version 3.02 for data clearance and exported to SPSS version 20 for analysis. AOR with $95 \%$ C.I results of multivariate logistic regression was used to assess the statistical significance of associations between independent and dependent variables. The level of statistical significance was declared at $p<0.05$.

Result: The median calcium intake was $543 \mathrm{mg}$ per day. From 398 participants, 265(66.6\%) were at risk of inadequate intake of calcium. Greater than half 249 (62.6\%) respondents had practiced avoiding calcium rich food during their pregnancy. The finding of this study identified that nutritional counseling have strong statistical association with dietary calcium intake of mothers during pregnancy. The chance of dietary calcium intakes during pregnancy who were received nutritional counseling 2.4 times higher than those who were not received nutritional counseling (AOR=2.432 95\% Cl: 1.072-5.517).

Conclusion and recommendation: From the present study, it can be concluded that, majority of pregnant mothers $265(66.6 \%)$ had a poor dietary calcium intakes during their pregnancy. It is recommended that consumption of enough calcium and dairy products should be included and emphasized in the nutrition education component of maternal health programs.

\section{Introduction}

Inadequate intakes of calcium are one of the most serious public health problem affecting mothers and their children in the world. WHO estimates that at least one woman dies every seven minutes from complications of hypertensive disorders of pregnancy this is may be because of low intakes of dietary calcium [1]. 
Inadequate intakes of calcium during pregnancy cause hypertensive disorder which is a major health problem leading maternal and perinatal morbidity and mortality. However, an adequate calcium intake during pregnancy might prevent pregnancy induced hypertension, preterm birth, FGR, increasing maternal bone mass, and reduce fracture risks [2-3].

In resource-poor environments across the globe, low quality monotonous diets are the norm. When grainor tuber-based staple foods dominate and diets lack vegetables, fruits and animal-source foods, risk for a range of micronutrient (calcium) deficiencies is high. Mainly pregnant women is vulnerable group, but there is no sufficient information on micronutrient (calcium) deficiencies on such global public health problem among pregnant women [4].

There is less evidence from developing countries generally and specifically where monotonous diets, relying mostly on a typical few plant-based staple foods confirm this association specifically among pregnant women [5]. Maternal diets during pregnancy need to provide nutrients for the mother as well as for fetal growth and development. Prevention of Hypertensive disorders and preeclampsia are the advantages of dietary calcium intakes during pregnancy[6]. Ensuring that appropriate dietary calcium is consumed is important for preventing pregnancy induced hypertension and helping to reduce the risk of osteoporosis with advancing age [7-8].

So evaluation of dietary calcium intake and associated factors among pregnant women in Loma Woreda, Dawuro, South West Ethiopia is very important.

\section{Materials And Methods}

\section{Study Settings and study period}

The study was conducted in Loma Woreda from May to July 2019 which is located $512 \mathrm{~km}$ away from Addis Ababa, the capital city of Ethiopia, $292 \mathrm{~km}$ from Hawassa, the regional town SNNPR. The district structured in to 24 rural and 3 urban kebeles. According to the report of CSA the estimated population of Loma Woreda was 108,965 out of which 53,502 were male \& 55,463were female[9]. Maize, enset (false banana), and root crops are majorly consumed food items [10].

Study Design: Community based cross sectional study design was used.

Source Population: All pregnant women who are living at least for six months in Loma Woreda

Study Population: Randomly selected pregnant women were participated during the study period.

Inclusion criteria: - Pregnant women who are living at least for 6 months in Loma Woreda.

Exclusion criteria: Seriously ill pregnant women during the interview were not considered in study.

Sample size determination 
The sample size was determined by using single proportion formula by taking assumptions of the prevalence of dietary calcium intake as $50 \%, 5 \%$ marginal error, $95 \% \mathrm{Cl}$, a non-response rate of $10 \%$. Therefore, sample size is: $n=(z a / 2) 2 p(1-p) / d 2=(P=50 \%)(1.96)^{2 \star 0.5}(1-0.5) /(0.05)^{2} n=384$

When adding $10 \%$ none response rate, the maximum sample size were $(384 * 10 \%)=422$.

\section{Sampling procedures}

From the total of 27 kebeles of Loma Woreda, 9 kebeles was selected by simple random sampling method. A sample frame from selected kebeles was taken from kebele health posts. Sample size for each selected kebeles was allocated proportionally. Study participants were selected by using simple random sampling technique. List of participating households from the selected kebeles was obtained from HEW. After obtaining a list of participant households, we have selected the required sample size by using lottery methods. A pregnant woman in each 9 kebeles and the first household was selected by simple random sampling method.

\section{Operational definitions}

Above RDA: The amount calcium intake is above1000 mg per day.

Below RDA: The amount of calcium intake is less than 1000 mg per day.

Adequate Intake: The amount of calcium intake is equals to $1000 \mathrm{mg}$ per day.

Positive Attitude: Pregnant women's score is $\geq$ the mean score 25 in likert scale

Negative Attitude: Pregnant women's score is $<25$ in likert scale.

Adequate Knowledge: Pregnant women score is $\geq$ the average score 7

Poor knowledge: Pregnant women score is < average score 7

\section{Data collection tools and procedure}

\section{Recruitment of data collectors}

Eight diploma nurses were recruited to collect the data and one BSC nurse and one Health officer were supervise data collection. The data collector and supervisor were trained for two consecutive days on the objective, ethics, method and contents of question.

\section{Quantified multiple pass $24 \mathrm{hr}$ Recall}

The 24 hour dietary recall includes the type of food and beverages consumed inside and outside of the house along with the quantity of the foods. The 24-hour period starts with the first food eaten by the pregnant women in the morning until the last food item consumed before going to bed. The Quantified 24 hr recall was used for at least three different days which covers at least one week day and one weekend 
day so as to adequately represent the habitual intake. The FAO (2018) Quick guide to use $24 \mathrm{hr}$ recall was followed.

Dietary Recall: Each interview involved, a stepwise series of questions, normal household utensils, food substitutes (play dough, flour, lentils, water) and pictures of most commonly consumed foods in the specific regions to improve the memory of the respondents and assist in completing the questionnaires.

$24 \mathrm{hr}$ dietary recall was conducted using the multiple-pass technique which include,

1. First pass:-Quick list of all foods, drinks, snacks consumed from midnight of one day until midnight the following day.

2. Second pass:-Detailed and more specific descriptions of all the foods and drinks consumed, including cooking methods and brand names (where possible). The interviewer also asked if the respondent has remembered any additional items that were consumed but which were forgotten in the first pass.

\section{Third pass:-Estimating portion sizes}

In order to accurately estimate the portion size of foods and drinks consumed, Calibrated locally available labeled cup, bowl, spoon, serving spoons, food weighing scale. Moreover, the data collectors carried different sizes of locally consumed fruits and vegetables such as bananas, avocado, shallot, tomato, potato, yum to facilitate the recall [21].

4. Pass four:-The interviewer reviewed the recall to ensure that all the items have been recorded correctly. Every respondent were probed for snacks, drinks and outdoor consumption of any kind of food after they complete recall.

The interviews were conducted on all seven days of the week to capture changes in intakes across various days of the week. The dietary recall was repeated in randomly selected of the households by interviewer and in a different nonconsecutive day than the first. The recall was repeated to adjust for the day to day variation we were include week day and weekends and to adjust within person variation of nutrient intakes of the study participants we were increased days. All recall days were arranged on nonspecial occasion days like holidays or feasts.

The calcium intake was calculated by using food composition table of Ethiopia[22]. After calculating the intake of dietary Calcium per day the values was compared with WHO Recommended Dietary Allowances of Calcium for pregnant women as there is a lack of national standards.

\section{The standard Questionnaire}

Data was collected by using structured questionnaire which is adopted after reviewing literatures of similar studies based on the objective of this study. An interactive, multiple-pass $24 \mathrm{hr}$ dietary recall questionnaire adapted and validated for use in developing countries [30] was used to collect data on dietary intake. The knowledge and attitude of pregnant was categorized on two and five-level which 
includes the responses for given negative and positive statement as correct and incorrect and strongly disagree, disagree, neither agree nor disagree, strongly agree, agree and $(1,2,3,4,5)$ of likert scale.

\section{Data quality assurance}

Before data collection, the study questionnaire was checked using a pretest of $5 \%$ of invited pregnant women from households. Before estimating portion size we were standardizes measuring tools, and we were observed locally available measuring tools in study area then measure the common foods from 10(ten) household by digital food scale used to measure the gram amount of food consumed and of ingredients used in food preparation and finally took the average amount. Drinking cups glasses, spoons and ladles were labeled in to three categories based on their size (large, medium and small).

The questionnaire was prepared initially in English and then translated into Amharic Language, then retranslated back to English by another person who has good command of English, Amharic language to check for its consistency. The data collectors and supervisors were trained two days particularly in the proper filling of multiple pass 24 hour recalls. Information on 24-hour recall is collected using an openended format. Quantitative information on food intake, as described using portion size, allows for the calculation of calcium intakes of a 24-hour recall form. Estimation of portion size is facilitated by the use of measurement aids such as standard household measurement tools [11]. To calculate calcium intakes, the estimated portion size or the amount of food intake was multiplied by the values of nutrient content in foods as found in the Ethiopian food composition tables[22].

\section{Data management and analysis plan}

Data was checked for completeness and consistency, edited manually, coded and then entered in to Epi Data version 3.02 for data clearance and exported into SPSS version 20 for analysis. Descriptive statistics was used to calculate frequencies and percentages of study participants with regard to different variables. Variables with a p-value less than 0.25 in the bi variable logistic regression analysis were candidate for multivariable analysis. AOR with $95 \%$ C.I. of $p<0.05$ in multivariable logistic regression was considered as statistically significant.

Ethical clearance was obtained from Ethical Review Committee (ERC) of Wolaita Sodo University, department of Human Nutrition and support letter was to Loma Woreda Health office.

\section{Result}

\section{Socio-demographic and Economic Characteristics of Participants}

Three hundred and ninety eight (398) subjects were participated in the study with response rate of $94 \%$. The median age of study subjects was 30 years. Concerning educational level, 176 (44.2\%) of the respondents were illiterate and 365(91.7\%) of pregnant women's earned less than 1000 ETB per month 
monthly income. Two hundred twenty seven $(57 \%)$ of the respondents had nutritional information during their pregnancy. 
Table 1

Socio-demographic and economic factors among pregnant women in Loma woreda, south west, Ethiopia $(n=398)$

\begin{tabular}{|ll|}
\hline Characteristics & Numbers (\%) \\
\hline Age group of subject & \\
\hline $15-24$ & $56(14.1)$ \\
\hline $25-34$ & $254(63.8)$ \\
\hline $35-44$ & $88(22.1)$ \\
\hline Monthly income in ETB & \\
\hline$<1000 E T B$ & $365(91.7)$ \\
\hline $1000-2000 E T B$ & $17(4.3)$ \\
\hline$>2000 E T B$ & $16(4)$ \\
\hline Marital Status & \\
\hline Married & $357(89.7)$ \\
\hline Single & $2(0.5)$ \\
\hline Divorced & $11(2.8)$ \\
\hline Widowed & $28(2)$ \\
\hline Occupational status & \\
\hline Farmer & $87(21.9)$ \\
\hline Housewife & $281(70.6)$ \\
\hline Civil servant & $22(5.5)$ \\
\hline Merchants & $8(2.2)$ \\
\hline Household size & $176(26.6)$ \\
\hline$<4$ & $191(48)$ \\
\hline$>=4$ & $207(52)$ \\
\hline Education & \\
\hline Illiterate & \\
\hline Primary & \\
\hline Secondary \& above & \\
\hline Market Availability & \\
\hline
\end{tabular}




\begin{tabular}{|ll|}
\hline Characteristics & Numbers (\%) \\
\hline Yes & $118(29.6)$ \\
\hline No & $280(70.4)$ \\
\hline
\end{tabular}

\section{Calcium Intakes}

Out of 398 study participants responded for the questions to assess their dietary calcium intakes, $66.6 \%$ of them had inadequate calcium intakes in which, greater than half 249 (62.6\%) respondents had practiced avoiding calcium rich food during their pregnancy. Out of those who avoided food during their pregnancy, due to it makes the baby big, culture and it makes delivery difficult. About $44.7 \%$ pregnant women of this study were agreed on satisfactory birth out comes can be achieved through nutrition knowledge and practice of pregnant women.

The median intake was $543 \mathrm{mg} /$ day. The Proportion of pregnant women with low calcium intakes (less than $1000 \mathrm{mg}$ per day) was 265(66.6\%).

\section{Meal consumption pattern of pregnant women}

Three hundred eighty three (96.2\%) of the respondents had not practiced the habit of eating snacks between meals during their pregnancy. Majority of the pregnant mothers do not consume their snacks.

\section{Group of Foods consumed by pregnant women}

Plant based foods such as cereal, roots and tubers and also green leafy vegetables were the most consumed. Flesh foods and milk products were the least consumed food groups.

\section{Dietary Diversity Score of pregnant women}

Out of 398 study participants responded for the questions to assess their dietary diversity, 241(61\%) of them had inadequate dietary diversities. Different evidence showed that in developed countries, dietary diversity is strongly associated with nutrient adequacy [20].

\section{Factors associated with calcium intake}

Age, level of education, distance from health facility, occupation, household size, nutritional counseling, regular milk consumption, importance of calcium, culture and facility visit were the factors associated with dietary intake of calcium.

Multivariate analysis showed that there was statistically significant association between occupation and dietary calcium intakes of mothers during pregnancy. Women who were being civil servant (AOR 0.052 $95 \% \mathrm{Cl} 0.01-0.269)$ were less likely to have calcium intakes than farmers. The chance of dietary calcium 
intakes during pregnancy among mothers who were received nutritional counseling 2 times more likely than those who were not received nutritional counseling(AOR $=2.43295 \% \mathrm{Cl}: 1.072-5.517)$.

Relative to the pregnant women who were agreed on calcium importance had significantly 3 times more likely to had good dietary calcium intakes during their pregnancy ( $\mathrm{AOR}=3.256,95 \% \mathrm{Cl} ; 1.241-8.547)$ than women who were not agreed on calcium importance. 
Table 2

Dietary calcium intake and socio-demographic characteristics in pregnant women in Loma woreda southern Ethiopia 2019 (Bivariate analysis)

\begin{tabular}{|c|c|c|c|c|c|}
\hline \multirow[b]{3}{*}{ Variables } & \multicolumn{5}{|c|}{ Calcium intakes } \\
\hline & \multicolumn{2}{|c|}{$<$ Median value } & \multicolumn{2}{|c|}{ >= Median value } & \multirow[t]{2}{*}{ p-value } \\
\hline & $\mathrm{N}$ & $\%$ & $\mathrm{~N}$ & $\%$ & \\
\hline \multicolumn{6}{|l|}{ Age } \\
\hline $15-24$ & 29 & 7 & 27 & 7 & \multirow[t]{3}{*}{0.023} \\
\hline $25-34$ & 125 & 31 & 129 & 32 & \\
\hline $35-44$ & 47 & 12 & 41 & 10 & \\
\hline \multicolumn{6}{|l|}{ Education } \\
\hline No education & 94 & 24 & 82 & 21 & \multirow[t]{3}{*}{$<0.001$} \\
\hline Primary & 67 & 17 & 39 & 10 & \\
\hline Secondary and above & 40 & 10 & 76 & 19 & \\
\hline \multicolumn{6}{|c|}{ Distance from health facility } \\
\hline$<=10 \mathrm{~km}$ & 94 & 24 & 112 & 28 & \multirow[t]{2}{*}{0.001} \\
\hline$>=11 \mathrm{~km}$ & 107 & 27 & 85 & 21 & \\
\hline \multicolumn{6}{|l|}{ Visit to PHCU } \\
\hline Yes & 106 & 27 & 163 & 41 & \multirow[t]{2}{*}{0.001} \\
\hline No & 95 & 24 & 34 & 8 & \\
\hline \multicolumn{6}{|c|}{ Nutritional counseling received } \\
\hline Yes & 78 & 20 & 149 & 37 & \multirow[t]{2}{*}{$<0.001$} \\
\hline No & 123 & 31 & 48 & 12 & \\
\hline \multicolumn{6}{|c|}{ Regular consumption of milk and milk products is good for pregnant women } \\
\hline Agree & 46 & 12 & 119 & 30 & \multirow[t]{2}{*}{$<0.001$} \\
\hline Disagree & 155 & 39 & 78 & 19 & \\
\hline \multicolumn{6}{|c|}{ Regular intake of green leafy vegetables is good for pregnant women } \\
\hline Agree & 74 & 18 & 127 & 32 & \multirow[t]{2}{*}{$<0.001$} \\
\hline Disagree & 127 & 32 & 70 & 18 & \\
\hline
\end{tabular}




\begin{tabular}{|c|c|c|c|c|c|}
\hline \multirow[b]{2}{*}{ Agree } & \multicolumn{5}{|c|}{ Calcium intakes } \\
\hline & 54 & 13 & 124 & 31 & \multirow[t]{2}{*}{$<0.001$} \\
\hline Disagree & 147 & 37 & 73 & 18 & \\
\hline \multicolumn{6}{|c|}{ Calcium is important for the pregnant women and growing fetus } \\
\hline Agree & 28 & 7 & 83 & 21 & \multirow[t]{2}{*}{$<0.001$} \\
\hline Disagree & 173 & 43 & 114 & 29 & \\
\hline \multicolumn{6}{|c|}{ My culture encourages me to take milk and dairy products, green leafy vegetables during pregnanc } \\
\hline Agree & 64 & 16 & 85 & 21 & \multirow[t]{2}{*}{0.014} \\
\hline Disagree & 137 & 34 & 112 & 28 & \\
\hline \multicolumn{6}{|c|}{ Awareness on recommended dietary intake during pregnancy reached rural areas. } \\
\hline Agree & 137 & 34 & 104 & 27 & \multirow[t]{2}{*}{$<0.001$} \\
\hline Disagree & 64 & 16 & 93 & 23 & \\
\hline \multicolumn{6}{|c|}{ Dietary calcium intakes during pregnancy is bad } \\
\hline Agree & 168 & 42 & 118 & 30 & \multirow[t]{2}{*}{$<0.001$} \\
\hline Disagree & 33 & 8 & 79 & 20 & \\
\hline \multicolumn{6}{|l|}{ Kind of services } \\
\hline No visit & 95 & 24 & 34 & 9 & \multirow[t]{3}{*}{$<0.001$} \\
\hline ANC visit & 95 & 24 & 148 & 36 & \\
\hline Other visit & 11 & 3 & 15 & 4 & \\
\hline \multicolumn{6}{|l|}{ Family size } \\
\hline$<4$ & 90 & 23 & 101 & 25 & \multirow[t]{2}{*}{0.083} \\
\hline$>=4$ & 111 & 28 & 96 & 24 & \\
\hline \multicolumn{6}{|c|}{ Knowledge on dietary calcium intakes } \\
\hline Adequate & 101 & 25 & 69 & 17 & \multirow[t]{2}{*}{0.036} \\
\hline Poor & 100 & 25 & 128 & 33 & \\
\hline \multicolumn{6}{|c|}{ Attitude towards dietary calcium intakes } \\
\hline Positive attitude & 103 & 26 & 75 & 19 & \multirow[t]{2}{*}{0.043} \\
\hline Negative Attitude & 98 & 25 & 122 & 30 & \\
\hline
\end{tabular}




\begin{tabular}{|llllll|}
\hline \multicolumn{7}{l|}{ Calcium intakes } & & & \\
\hline Adequate & 61 & 15 & 96 & 24 & 0.105 \\
\hline Inadequate & 136 & 35 & 105 & 26 & \\
\cline { 1 - 3 } & & & & & \\
Yes & 70 & 18 & 48 & 12 & 0.303 \\
No & 131 & 33 & 149 & 37 & \\
\hline
\end{tabular}


Dietary calcium intake and associated factors in pregnant women in Loma woreda, south west Ethiopia, 2019 (multivariate logistic regression).

\begin{tabular}{|c|c|c|c|c|c|}
\hline \multirow[t]{2}{*}{ S/no } & \multirow[t]{2}{*}{ Variables } & \multirow[t]{2}{*}{$N(\%)$} & \multicolumn{3}{|c|}{$\begin{array}{l}\text { Daily dietary calcium intake } \geq \text { median } \\
\text { value }\end{array}$} \\
\hline & & & OR & $95 \% \mathrm{Cl}$ & p-value \\
\hline \multirow[t]{5}{*}{1} & Occupation of the respondents & & & & \\
\hline & Civil servants & $22(5.5)$ & 1 & 1 & \\
\hline & Housewives & $281(70.4$ & 0.207 & $0.066-0.654$ & 0.007 \\
\hline & Farmers & $87(21.8)$ & 0.052 & $0.01-0.269$ & 0.001 \\
\hline & Merchants & $8(2.3)$ & 0.023 & $0.002-0.279$ & 0.003 \\
\hline \multirow[t]{3}{*}{2} & Family size & & & & \\
\hline & $>4$ & $207(52.1)$ & 0.285 & $0.111-0.733$ & 0.009 \\
\hline & $<=4$ & 191(47.9) & 1 & 1 & \\
\hline
\end{tabular}

$3 \quad$ Attitude towards dietary calcium intake

Negative attitude

Positive attitude

$4 \quad$ Nutritional counseling received

No

171

(43.1)

Yes

(56.9)

Daily dietary calcium intake $\geq$ median 
In multivariate logistic regression, occupation $A O R=0.052(0.01-0.269)$, family size, $A O R=0.285(0.111-$ $0.733)$, attitude towards calcium intakes $A O R=0.003(0.001-0.016)$, nutritional counseling $A O R=2.432$ (1.072-5.517), knowledge on calcium AOR 3.256 (1.241-8.547), regular consumption of milk AOR = $2.806(1.124-7.005)$ and age of respondents $A O R=0.126(0.027-0.581)$ are associated factors with dietary calcium intakes among pregnant women.

\section{Discussion}

The study was inspected the dietary calcium intakes and associated factors among pregnant women. Low dietary Calcium intake was widespread among pregnant women. Findings of this cross-sectional study indicate that a high proportion of pregnant women in study area do not consume enough calcium as well as calcium-rich food products; and meeting adequate intake of this nutrient is not possible through the current dietary patterns in the majority of the studied population. Dietary calcium intake was associated with age, occupation of respondents, family size, nutritional counseling, attitude towards dietary calcium intake and knowledge on calcium importance and regular consumption of milk.

\section{Dietary Calcium Intake}

The study presented that the average calcium intake in pregnant women in our study area was low (543 mg/day) compared to WHO recommendation. This result clearly reflects the low consumption of calcium-rich products and culture of the community. The finding was correlated with survey conducted in china and Iran mean intakes were 633.2 \pm 492.4 and $644 \pm 255$ respectively in 2015[13]. Likewise, low calcium intakes (561 mg/day) were observed in Benin 2014 [14].Researcher's also reported similar finding on the pregnant women in the study conducted in Sidama Zone, Southern Ethiopia. As it was observed with the other micronutrients, calcium intake was also lower than the recommendation in both of the study groups. Its intake was much lower (below half of the requirement) during their pregnancy [15].

In differently, higher dietary calcium intakes $1,256.9 \mathrm{mg} /$ day were observed in Thailand 2018[16].

Consequently to low average calcium intakes, the occurrence of adequate calcium intake in pregnant women in the current study is low. $33.4 \%$ of pregnant women have adequate calcium intake. This result is comparable in Nigeria the prevalence of calcium intake is $40 \%$ [10].

In contrast, higher prevalence of adequate calcium intake in America and Canada, and Europe, with their mean daily calcium intake as 1176 , and $1141 \mathrm{mg} /$ day respectively $[6,17]$. The discrepancy of the two studies may be due to the fact that the differences between the study participants, in that the present study was conducted on rural communities ( pregnant mothers) which can be lower than rural mothers in terms of educational, economic status and more access to nutrition information during pregnancy. 
The different results underlined differences in dietary consumptions. In developing country especially in our study area dietary calcium intakes among pregnant women are very low for the reason that diets are mainly on cereal and legumes based $[18,8,19]$. In addition the culture of community were prohibited to consume calcium rich food products for pregnant women mainly milk and milk products because of this food groups fattening the fetus and difficulty happen during delivery time. Whereas, in developed country, calcium intake is high due to production and usual consumption of dairy products. Definitely, consumption of calcium-rich foods is low in the study population [19].

Meaningful that hypertensive conditions and its difficulties during gestation are prevalent in developing countries and the positive role of adequate calcium intake in reducing the risk of hypertensive disorders, the common inadequate calcium intake through pregnancy in developing countries requires adequate interventions [7].

\section{Factors Associated with Dietary Calcium Intake in Pregnant Women}

Women who were being civil servants (AOR $0.05295 \% \mathrm{Cl} 0.01-0.269$ ) were less likely to have calcium intakes than farmers. This result is supported by the study conducted in Kenya in which employee was associated with dietary calcium intakes (AOR $=1.0895 \% \mathrm{Cl} 0.88-2.45)$.

Also in this study women's dietary attitude had shown negative relationship with dietary calcium intake of mothers during pregnancy $(A O R=0.00395 \% \mathrm{Cl} 0.001-0.016)$. This finding is also in agreement with the study conducted in America in which the most significant predictor for good dietary practice was women's attitude towards dietary intake of calcium ( $A O R=2.32,1.56-3.43)$.

The present study also demonstrated that there was strong statistical association between regular consumption of milk and milk products and dietary calcium intake during pregnancy (AOR $=3.25695 \% \mathrm{Cl}$ $1.241-8.547)$. This result is supported by the study conducted in Thailand in which consumption of milk products was significantly associated with dietary calcium intakes (3.9 95\% Cl: 2.0-5.9)[16]

The finding of this study identified that nutritional counseling have strong statistical association with dietary calcium intake of mothers during pregnancy AOR $=2.43295 \% \mathrm{Cl}: 1.072-5.517$ ). This result is supported by the study conducted in Thailand in which nutrition information was the predictor factor for dietary calcium intakes in the study area $(\mathrm{AOR}=0.985,95 \% \mathrm{Cl}$ : $0.775-1.250)$.

Pregnant women in urban areas, women with those whose household size is less than four were significantly less likely to have higher calcium intakes compared to rural women, with those whose household's size was four or less(AOR $0.28595 \% \mathrm{Cl} 0.111-0.733$ ). This result is supported by the study conducted in Benin in which house hold size was significantly associated with dietary calcium intakes (AOR 1.330 95\% Cl 1.092-1.618)[19]

\section{Conclusion}


From the present study, it can be concluded that, majority of pregnant mothers (66.6\%) had a poor dietary calcium intakes during pregnancy. They lacked the basic and the essential foods to consume vegetables, fruits, egg, milk and others which are the basic sources of most of calcium. Moreover, unhealthy dietary intake was observed among them.

The current study also showed that, the most significant predicting factors for dietary calcium intakes in this study were women's nutrition knowledge, regular consumption of milk, importance of calcium, age and family size.

Study results show that dietary calcium intakes of pregnant women in the study area were not adequate. The results suggest that pregnant women need nutrition counseling regarding calcium rich food intake practices during pregnancy.

\section{Abbreviations}

$\mathrm{Al}=$ Adequate Intake

AOR=Adjusted Odd Ratio

$\mathrm{Ca}=$ Calcium

COR $=$ Crude Odd Ratio

$\mathrm{CSA}=$ Central Statics Agency

$\mathrm{FAO}=$ Food and Agriculture Organization

FNB=Food and Nutrition Book

RDA $=$ Recommended Daily Allowance

SNNPR= Southern Nation Nationalities Peoples Region

WHO $=$ World Health Organization

\section{Declarations}

By my signature below, I declare and confirm that this manuscript of research is our work. We have followed all ethical principles of research in the preparation, data collection, processing, analysis and completion of this thesis. All scholar materials that are included have been given recognition through citation. We affirm that we have cited and referenced all sources used in this document. Every serious effort has been made to avoid any plagiarism in the preparation of this manuscript. I really declare that this thesis is not submitted to any other institution anywhere for the publication. 
Sincerely

Adisu Hailu Tofu

\section{Consent form}

I Adisu Hailu Tofu give my consent for information about myself/my relative to be published in Nutrition \& Metabolism of BMC with manuscript ID- number - NUAM-D-20-00426,co-authors - Yibeltal Kassa and Damene Darota, I understand that the information will be published without my /my relative's as the name attached, but that full anonymity cannot be guaranteed. I understand that the text and any pictures or videos published in the article will be freely available on the internet and may be seen by the general public. The pictures, videos and text may also appear on other websites or in print, may be translated into other languages or used for commercial purposes. I have been offered the opportunity to read the manuscript.

\section{Competing interets}

The authors declare that they have no competing interest.

\section{Authors' Contributions}

AH was involved in proposal writing, designing, and recruitment and training of supervisors and data collectors, analysis and write-up and in all stages of the project implementation. She did most of the analysis and write up of the paper. YK contributed in the designing of the methodology, reviewing the proposal and paper and in the final approval of the paper. DD involved in designing of project proposal, design of questionnaires and in the final approval of the paper. All authors read and approved the final manuscript.

\section{Acknowlegements}

We would like to acknowledge Wolaita Sodo University; College of Medicine and Health Science for the approval of the ethical clearance. Our gratitude goes high school leaders, data collectors and students who participated in this study.

\section{Data Availability}

Data are available with the corresponding author in SPSS based.

\section{Ethical concerns}

Ethical clearance was taken from Ethical Review Committee of College of Medicine and health sciences of Wolaita Sodo University. Furthermore, letter of permission was obtained from Dawuro zone Health department. Consent was obtained from the study subjects whose age was 15 and above years after they 
were informed about objectives and procedures of the study and their right to refuse participation any time they want shall assured. No data will be shared to anyone to secure confidentiality.

\section{References}

1. Tranquilli AL, D.G., Magee L, Roberts J, Sibai BM, Steyn W, Zeeman GG, Brown MA, The classification, diagnosis and management of the hypertensive disorders of pregnancy. A revised statement from the ISSHP, 2014. 4: p. 97-104

2. Agriculture, U.S.D.o., Survey of Food Intakes by Individuals and Diet and Health Knowledge Survey. USDA's 2009: p. 3

3. Tesfaye A G, T.B.L., Sena B K, Pregnancy Induced Hypertension and Associated Factors among Pregnant Women Receiving Antenatal Care Service at Jimma Town Public Health Facilities, South West Ethiopia. J Gynecol Women's Health, 2018. 10(3).

4. Gynecologists, A.C.o.O.a., Hypertension in Pregnancy. Report of the ACOG 2013.

5. Lee SE, T.S., Merialdi M, Caulfield LE Dietary intakes of women during pregnancy in low-and middleincome countries. Public Health Nutr, 2012. 16(18): p. 14.

6. Bolland MJ, L.W., Tai V, et al, Calcium intake and risk of fracture. systematic review. BMJ., 2015. 351(h4580).

7. Reid IR, B.S., Bolland MJ, Calcium supplements: benefits and risks. J Intern Med, 2015. 278(4): p. 354-368.

8. Sukchan, P., Liabsuetrakul, T., Chongsuvivatwong, V., Songwathana, P., Sornsrivichai, V. and Kuning, M., Inadequacy of Nutrients Intake among Pregnant Women in the Deep South of Thailand. BMC Public Health, 2010. 10(572): p. 1471-2458.

9. Agency, E.C.S., Federal Democratic Republic of Ethiopia Central Statistical Agency Population Projection of Ethiopia for All Regions At Wereda Level. 2014-2017.

10. Profile, L.W.H.O.P., 2019.

11. FAO, Dietary Assessment: A resource guide to method selection and application in low resource settings. Rome. 2018.

12. (EHNRI), E.H.a.N.R.U., Food

13. Liu FL, Z.Y., Parés GV, Reidy KC, Zhao WZ, Zhao A, Chen C, Ning CY, Zheng YD, Wang PY., Nutrient Intakes of Pregnant Women and their Associated Factors in Eight Cities of China. Chin Med J 2015(128): p. 9.

14. Kumar, A., Devi, S.G., Batra, S., Singh, C. and Shukla, D.K. , Calcium Supplementation for the Prevention of Pre-Eclampsia. International Journal of Gynaecology and Obstetrics, 2009. 104:p. 4.

15. Yewelsew.Y, A.B., KMichael.H, Inadequate intake of dietary Zinc and calcium among pregnant women from subssistence householda in Sidamo, southern Ethiopia. Public Health Nutrition, 2007. 11(4): p. 8. 
16. Chotboon, C., Soontrapa, S., Buppasiri, P., Muktabhant, B., Kongwattanakul, K., \& Thinkhamrop, J, Adequacy of calcium intake during pregnancy in a tertiary care center in Thiland. International journal of women's health, 2018. 10: p. 5 ..

17. Ariyo, 0.0., Omolola. et al, Adequacy of nutrients intakes among pregnant Women in Ibadan, Nigeria. Journal of Applied and Environmental Sciences, 2010. 6: p. 4.

18. organization, W.h., Dietary Reference Intakes for Calcium, Phosphorus, Magnesium, Vitamin D and Fluoride. National Academy Press, Washington, DC, 2009.

19. WHO World Health Organization, G., World Health Report micronutrient 2010.

20. Martin-Prevel Y, A.M., Allemand P, Wiesmann D, Ballard TJ, Deitchler M, et al., Development of a dichotomous indicator for population-level assessment of the dietary diversity of women of reproductive age. 2017

21. Composition Table for use in Ethiopia. Part IV. EHNRI/Food Agriculture Organization, 1995-1997. Gibson RSF, E.L., An interactive 24-hour recall for assessing the adequacy of iron and zinc intakes in developing countries. HarvestPlus, 2008.

22. (EHNRI), E.H.a.N.R.U., Food

\section{Figures}




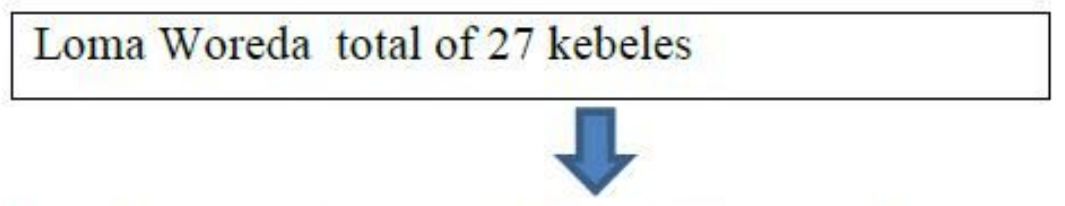

By simple random sampling method 9 kebeles was selected

\begin{tabular}{l|l|l|l|l|l|l|l|l|}
\hline $\mathrm{K} 1$ & $\mathrm{~K} 15$ & $\mathrm{~K} 2$ & $\mathrm{~K} 22$ & $\mathrm{~K} 3$ & $\mathrm{K25}$ & $\mathrm{K} 19$ & $\mathrm{~K} 10$ & $\mathrm{~K} 23$ \\
\hline $\mathrm{N}=\mathbf{6 2 4}$ & $\mathrm{N}=\mathbf{4 2 1}$ & $\mathrm{N}=\mathbf{5 8 8}$ & $\mathrm{N}=\mathbf{8 0 8}$ & $\mathrm{N}=\mathbf{3 5 1}$ & $\mathrm{N}=624$ & $\mathrm{~N}=617$ & $\mathrm{~N}=\mathbf{3 5 4}$ & $\mathrm{N}=\mathbf{6 2 6}$ \\
\hline
\end{tabular}

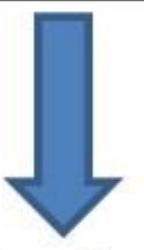

Proportionally allocated to the number of each selected kebeles using the formula

\begin{tabular}{|c|c|c|c|c|c|c|c|c|}
\hline K1 & K15 & K2 & K22 & K3 & K25 & K19 & K10 & K23 \\
\hline $\mathrm{n}=\mathbf{5 2}$ & $n=35$ & 49 & 67 & 30 & 54 & 52 & 30 & 53 \\
\hline
\end{tabular}

Figure 1

Schematic presentation of sampling procedure for the dietary calcium intakes and associated factors among pregnant women in Loma Woreda. 


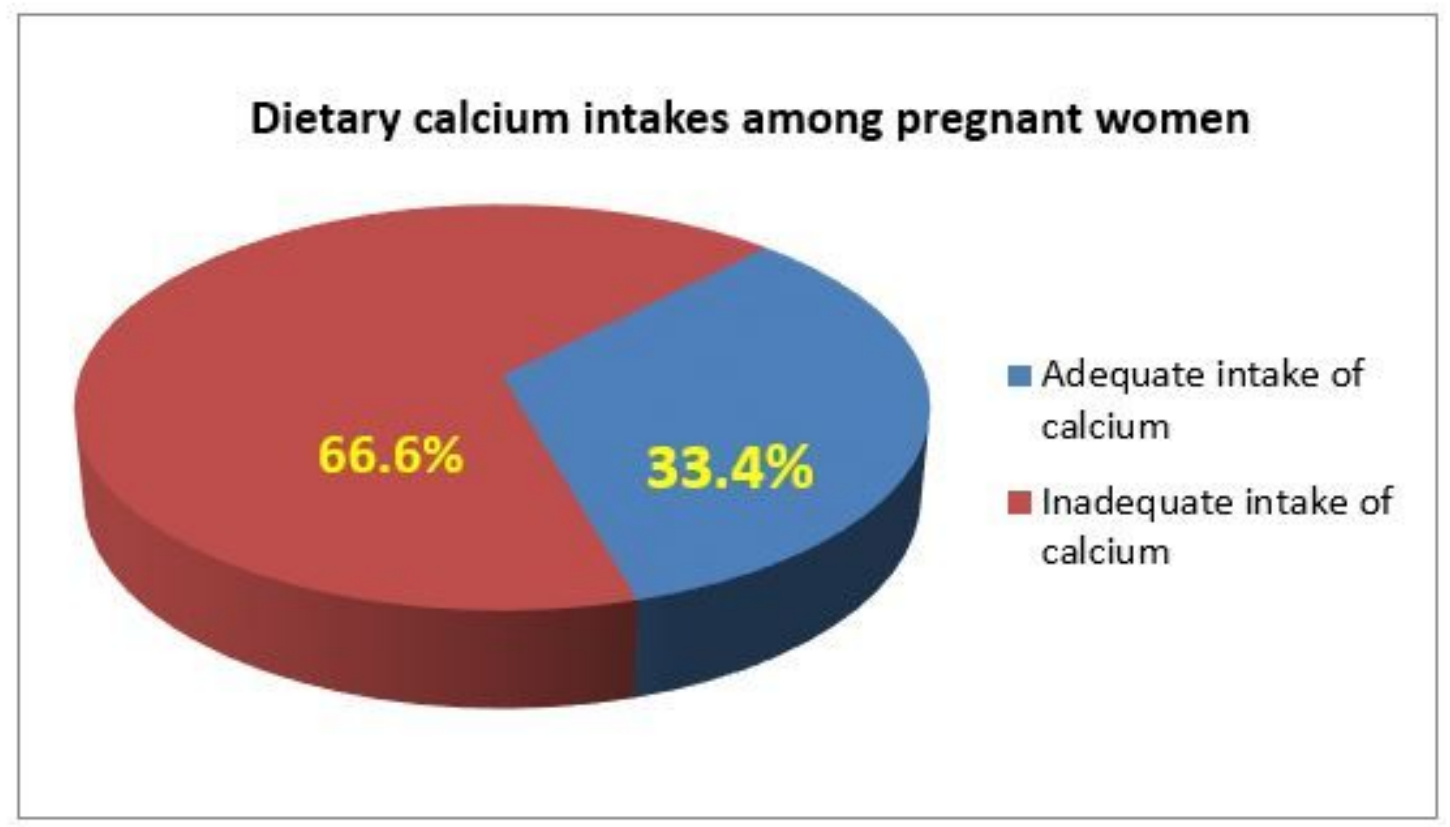

Figure 2

Distribution of dietary calcium intakes among pregnant women in Loma woreda, South west Ethiopia, $2019(n=398)$.

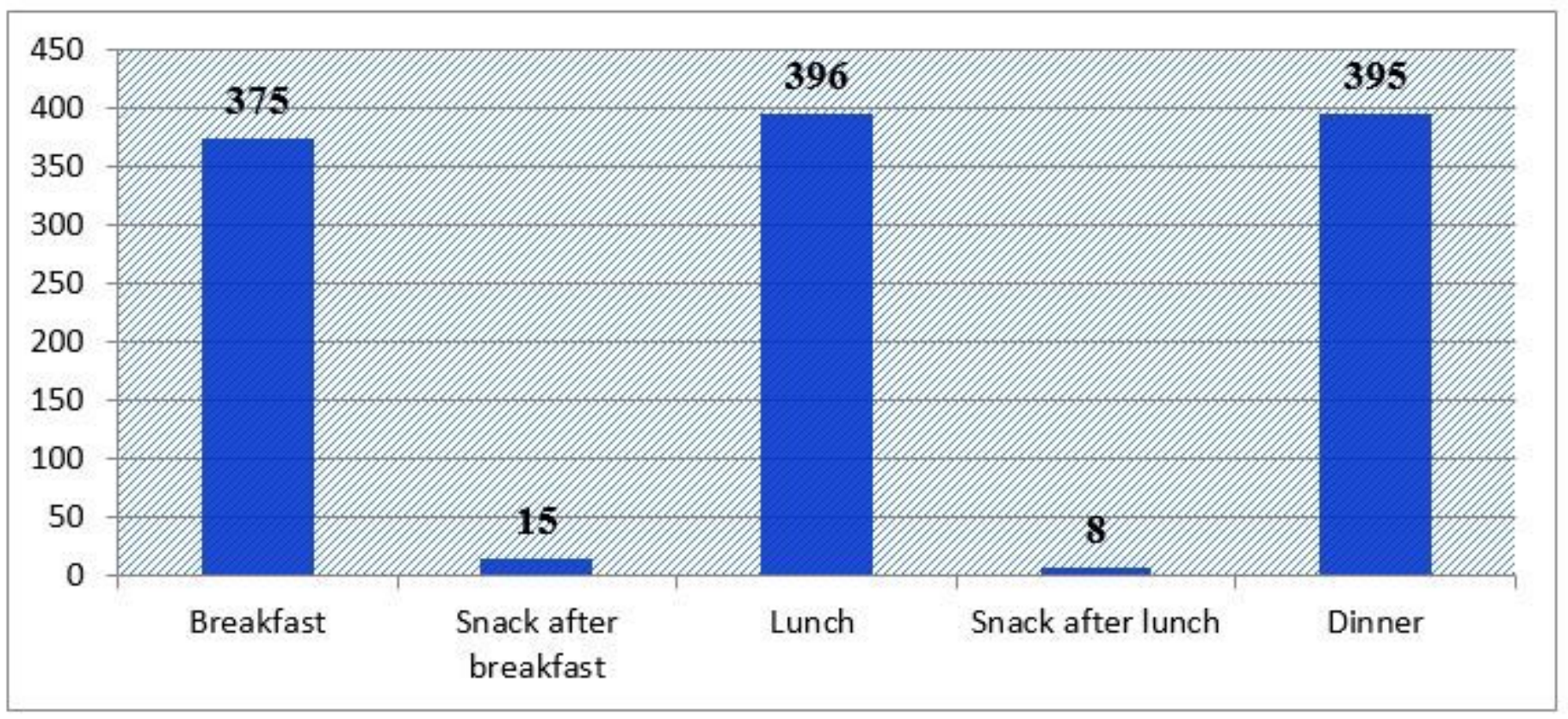

\section{Figure 3}

Meal pattern of pregnant women of Loma, south west Ethiopia, 2019(n=398) 


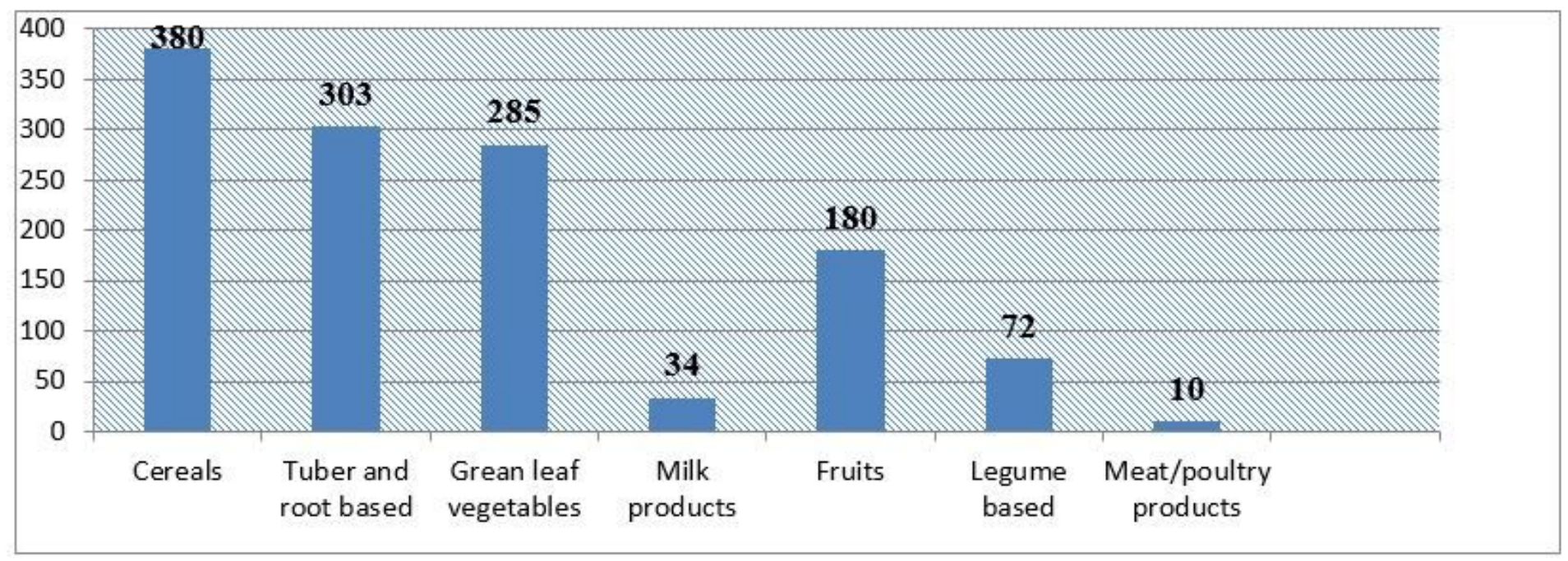

\section{Figure 4}

Proportion of food group consumed by pregnant women of Loma district, south west Ethiopia, $2019(n=398)$

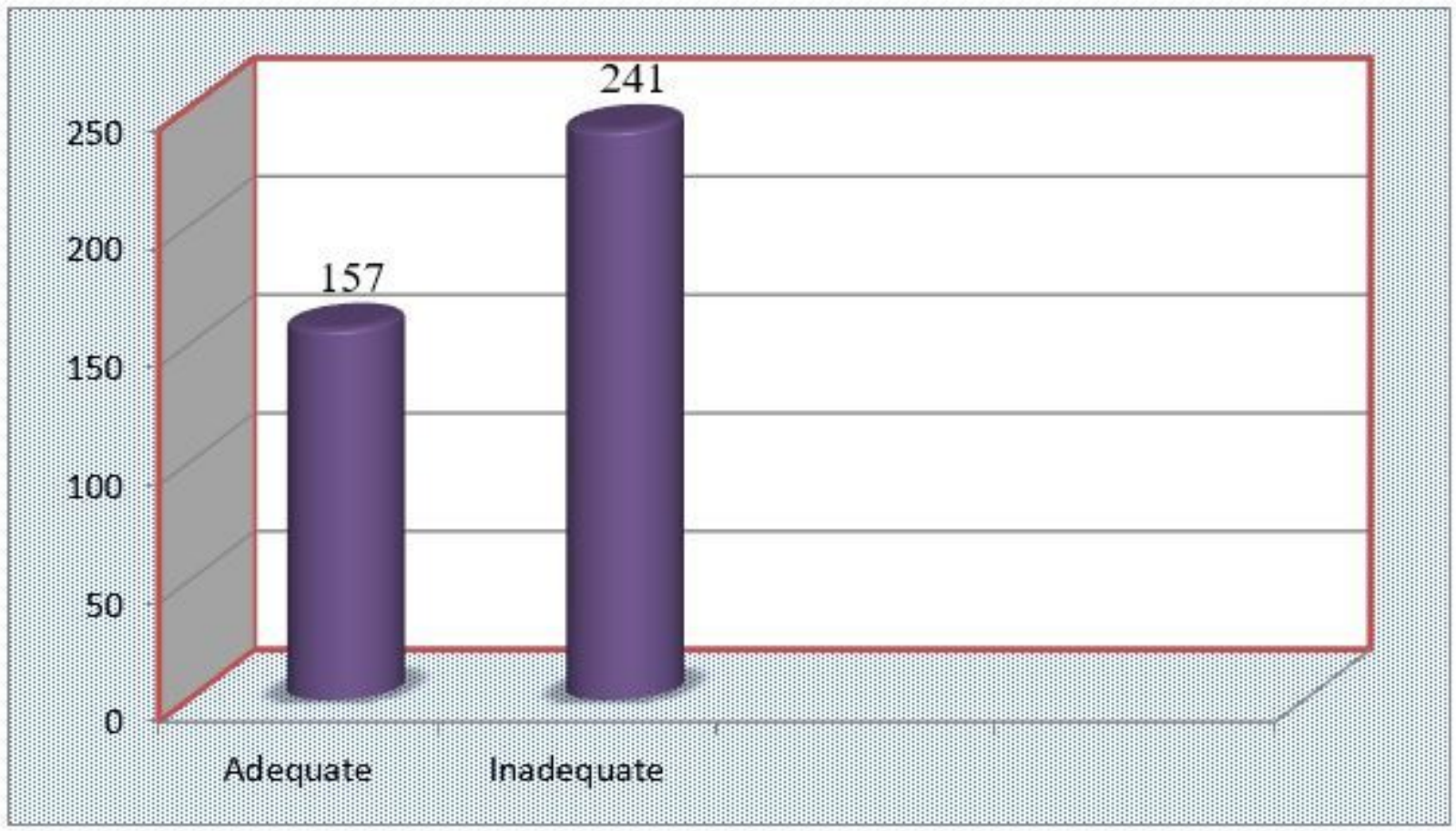

Figure 5

Dietary diversity score of pregnant women of Loma district, southwest Ethiopia, 2019 ( $n=398)$ 